JOURNAL

of Health Inequalities

\title{
Alcohol consumption trends and burden of alcohol- related health problems in Latvia
}

\author{
Laura Isajeva, Lauma Springe, Inese Gobina \\ Rīga Stradinš̌ University, Department of Public Healthand Epidemiology, Latvia
}

ADDRESS FOR CORRESPONDENCE: Laura Isajeva, Department of Public Health and Epidemiology,

Riga Stradinš University, Riga, Latvia; e-mail: laura.Isajeva@rsu.lv

Supplementary materials (slides from the conference) are available in Webbappendix at the Journal's website: https://www.termedia.pl/ Journal/Journal_of_Health_Inequalities-100

Alcohol use is a risk factor for many non-communicable diseases, accidents, violence, and suicides. Alcohol use was the sixth leading risk factor for death and disability in Latvia over the last decade. Since 2017 Latvia has had the highest alcohol consumption rate in Europe, which has continued to rise in recent years, resulting in a high disease burden. The analysis aims to compile alcohol-related public health indicators to provide an overview of alcohol consumption trends and the alcohol-related burden of health problems in Latvia.

Secondary research was used to review national alcohol-related indicators and make comparisons at the European level. The study compiles alcohol-related indicators from national and European reports/survey results, including disability-adjusted life years (DALYs), potential years of life lost (PYLLs), recorded alcohol consumption, the share of dependent drinkers, and heavy episodic drinking. The review spans the years 2010 to 2020 .

Latvia had the highest recorded alcohol consumption among the OECD countries, i.e. 12.9 litres of pure alcohol per capita (15+ years) in 2019. Over the last decade, alcohol consumption in Latvia was gradually increasing from 9.8 litres in 2010 and reaching its peak in 2018 with 13.2 litres.

Latvia had the highest share of alcohol-dependent drinkers among OECD countries - 10.4\% in 2016, almost three times more than the OECD average. The most proportion of alcohol-dependent drinkers are males. However, the latest results of the population survey showed a decrease in the share of dependent drinkers to $6 \%$ in 2020. The greatest decline was observed among males, from $19.4 \%$ in 2015 to $9.4 \%$ in 2020 .
However, the prevalence of heavy episodic drinking at least once a month over the last year among alcohol drinkers in Latvia was $14.2 \%$ in 2019 , which is below the EU average and is the lowest value along with Estonia among Baltic sea region countries.

In 2019 , there were 804.3 DALYs per 100,000 due to alcohol use disorders which are $37 \%$ more than the WHO European region average. Over the past ten years (20102019), the number of DALYs due to alcohol use disorders in Latvia has not changed significantly but remains high compared to other European region countries. DALYs due to alcohol use disorders for males were four times greater than for females (1350 vs. 339 years). In general, alcohol use disorders are the ninth leading cause of death and disability in Latvia.

Alcohol-related PYLL were the highest in Latvia among Baltic sea region countries (LV, LT, EST, PL) in 2017 (778 years), with the share of $10 \%$ of all PYLL causes.

While alcohol-related PYLL has remained relatively stable among Latvian men over the last decade but has decreased among females, it was still the highest among Baltic sea region countries in 2017 for both genders.

The alcohol-related burden of diseases in Latvia is one of the highest in Europe. The available data on alcohol use and alcohol-related health problems in Latvia indicate an urgent need for broader and more effective alcohol use prevention strategies and gender-specific programmes.

Key words: burden of disease, alcohol consumption, Latvia, heavy episodic drinking, DALY, PYLL.

\section{DISCLOSURE}

The authors report no conflict of interest. 